\title{
Vortex generation by deep-water breaking waves
}

\author{
N. E. Pizzo $\dagger$ and W. Kendall Melville \\ Scripps Institution of Oceanography, University of California, San Diego, La Jolla, CA 92093-0213, USA \\ (Received 14 August 2012; revised 15 July 2013; accepted 24 August 2013; \\ first published online 8 October 2013)
}

The connection between wave dissipation by breaking deep-water surface gravity waves and the resulting turbulence and mixing is crucial for an improved understanding of air-sea interaction processes. Starting with the ensemble-averaged Euler equations, governing the evolution of the mean flow, we model the forcing, associated with the breaking-induced Reynolds shear stresses, as a body force describing the bulk scale effects of a breaking deep-water surface gravity wave on the water column. From this, we derive an equation describing the generation of circulation, $\Gamma$, of the ensemble-average velocity field, due to the body force. By examining the relationship between a breaking wave and an impulsively forced fluid, we propose a functional form for the body force, allowing us to build upon the classical work on vortex ring phenomena to both quantify the circulation generated by a breaking wave and describe the vortex structure of the induced motion. Using scaling arguments, we show that $\Gamma=\alpha(h k)^{3 / 2} c^{3} / g$, where $(c, h, k)$ represent a characteristic speed, height and wavenumber of the breaking wave, respectively, $g$ is the acceleration due to gravity and $\alpha$ is a constant. This then allows us to find a direct relationship between the circulation and the wave energy dissipation rate per unit crest length due to breaking, $\epsilon_{l}$. Finally, we compare our model and the available experimental data.

Key words: air/sea interactions, vortex flows, wave breaking

\section{Introduction}

Breaking waves are an important mechanism for modulating and enhancing the flux of gases, heat and momentum across the air-sea interface (Melville 1996). Breaking is believed to be the main way that momentum is passed from the wind-driven irrotational wave field into the underlying rotational ocean currents. An improved knowledge of the modification of these fluxes due to breaking is crucial for a better understanding of the mechanisms involved in air-sea interaction, and specifically for the enhancement of coupled atmosphere-ocean models. Although there have been recent advances in laboratory and field measurements, the problems associated with wave breaking still present demanding experimental, numerical and theoretical challenges. Therefore, even simple theoretical models focusing on the response of the water column to breaking can prove to be valuable in gaining a better understanding 
of air-sea interaction from local to global scales (Banner \& Peregrine 1993; Melville 1996). In this paper, we present a model relating the circulation generated by a breaking wave to the wave energy dissipated due to breaking.

Duncan (1981) performed laboratory experiments involving quasi-steady breaking generated by flow over a submerged hydrofoil. Duncan's (1981) scaling of dissipation is consistent with Lighthill's (1978) description of the wave power generated by flow past a cylinder. Using the balance of forces for breaking waves presented by Duncan (1981), Phillips (1985) proposed a model for the energy dissipation rate of a breaking wave per unit length of breaking crest, $\epsilon_{l}$ :

$$
\epsilon_{l}=b \frac{\rho c^{5}}{g},
$$

where $\rho$ is the density of water, $g$ is the acceleration due to gravity, $c$ is the characteristic wave phase speed and $b$ is a non-dimensional breaking strength parameter.

Dimensional analysis arguments by Melville \& Rapp (1985), Melville (1994) and Drazen et al. (2008) show that $b$ is a function of the bandwidth of the breaking wave group, the rate at which focusing occurs, and the wave slope at breaking, $S$. Following Drazen et al. (2008), this is defined as the linear prediction of the slope at focusing of the wave packet,

$$
S=\sum_{n} a_{n} k_{n},
$$

where the summation is over all amplitudes $a_{n}$ and wavenumbers $k_{n}$ of the input wave packet.

Drazen et al. (2008) proposed an inertial scaling model for plunging quasi-twodimensional breaking based only on the local slope $h k$ at breaking, finding

$$
b=\beta(h k)^{5 / 2}
$$

where $\beta$ is a constant of order unity, $h$ is the wave height at breaking and $k$ is the characteristic wavenumber of the breaking wave defined through the linear dispersion relation, $c^{2}=g / k$. The slope at breaking $h k$ does not necessarily relate to $S$, the linear prediction of the slope at breaking, in a simple way, since wave breaking is an inherently nonlinear process, especially as the point of breaking is approached; however, the experiments and analysis of Drazen et al. (2008, §5) and Tian, Perlin \& Choi $(2010, \S 3.1 .5)$ show that within the scatter of the laboratory data the two quantities are approximately linearly related.

Equation (1.3) has been corroborated through analysis of laboratory experiments for plunging breaking waves by Drazen et al. (2008). Romero et al. (2012) showed (see figure 1) that when combined with a threshold for breaking, this power law extends to the data for the onset of breaking from Banner \& Peirson (2007); that is, it applies across the whole available range of breaking strength data.

Melville \& Rapp (1985) and Rapp \& Melville (1990) measured the loss of momentum and energy fluxes from surface waves due to breaking by using dispersive focusing of a wave packet to induce unsteady, quasi-two-dimensional breaking. In particular, Rapp \& Melville (1990, §5.4) found that for three different strengths of wave breaking (two of which were described as plunging and one as spilling) more than $90 \%$ of the energy lost by the wave field due to breaking was dissipated within four wave periods of the breaking event. Meanwhile, they found that between 58 and 


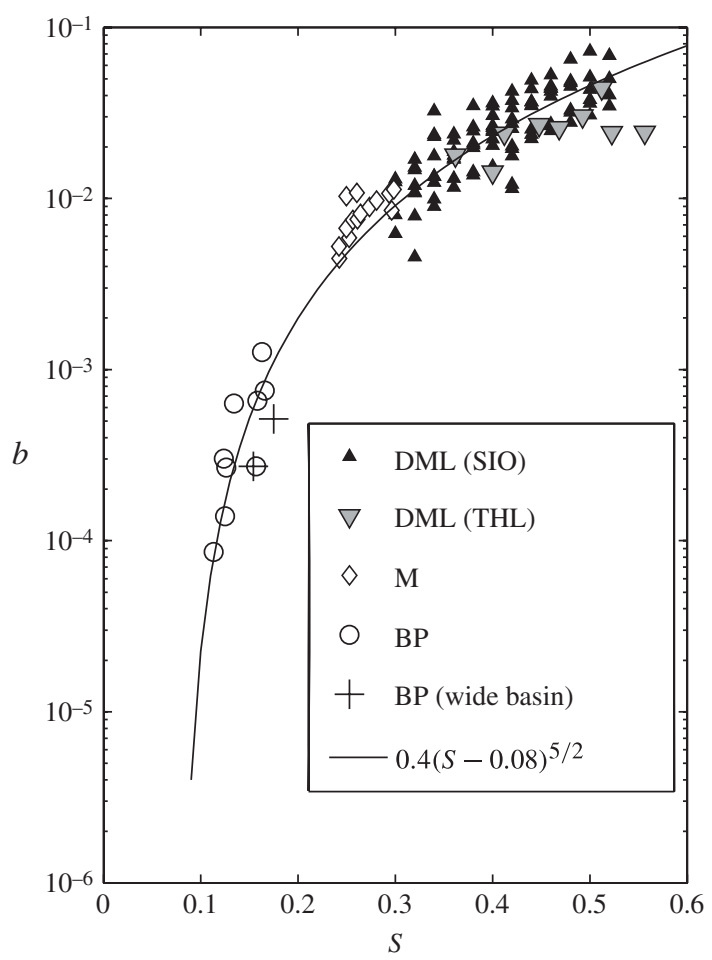

FIGURE 1. Analysis of laboratory measurements, adapted from Romero, Melville \& Kleiss (2012), of the breaking parameter $b$ versus the maximum linear slope at breaking, $S$, as defined in (1.2). DML (SIO) and DML (THL) are data from Drazen, Melville \& Lenain (2008), M are data from Melville (1994), BP and BP (wide basin) are data from Banner $\&$ Peirson (2007) and the solid line represents the fit predicted by the scaling argument of Drazen et al. (2008), with coefficient 0.4 and threshold slope of 0.08 chosen to best fit the data. See Romero et al. (2012) for further details.

$86 \%$ of the momentum lost by the wave field was present in the breaking-induced currents four wave periods after the breaking event. In addition, the authors found that the magnitudes of the induced mean and turbulent currents were comparable. Melville, Veron \& White (2002) (hereinafter referred to as MVW) used a similar dispersive focusing technique to study the ensemble averaged flow and turbulence after the breaking event. The post-breaking flow in these studies was dominated by a robust vortex, which is displayed in figure 2, lasting for more than 58 wave periods after breaking (see also Rapp \& Melville 1990, Sullivan et al. 2004). MVW showed that this vortex slowly propagated downstream under the influence of its image in the free surface.

To study the effects of breaking waves on the water column, Sullivan, McWilliams \& Melville (2004) (hereinafter referred to as SMM2004) proposed a heuristic model of a breaking wave by the use of a body force. The authors modelled this force based on laboratory experiments of Rapp \& Melville (1990) and MVW, finding qualitative and quantitative agreement between the large-scale response of the flow in the model and the (ensemble-averaged) behaviour observed in the laboratory. They then compared the 


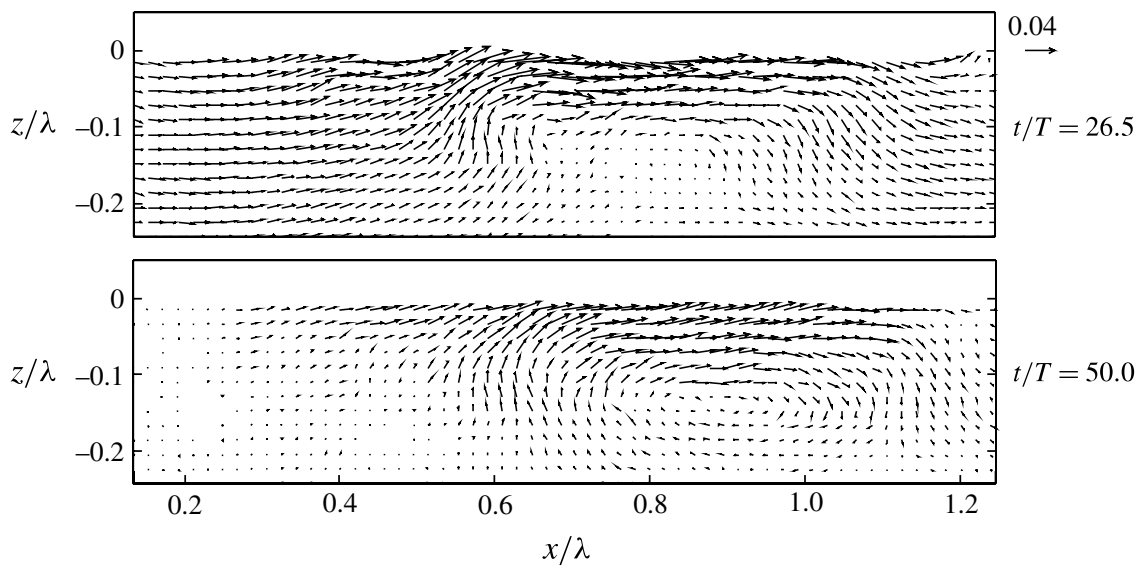

FIGURE 2. Laboratory measurements of the ensemble averaged, non-dimensionalized, velocity field induced by breaking, from Melville et al. (2002, MVW). Here, $\lambda$ and $T$ represent the characteristic wavelength and period of the breaking wave, respectively, and the vector density is reduced by a factor of 10 for clarity of presentation; see MVW for further details. The figure shown is for times $t / T=26.5,50$ for top and bottom plots, respectively. The main feature of the flow is a coherent vortex, which lasts for more than 50 wave periods after the breaking event and slowly propagates downstream through the action of its image in the free surface.
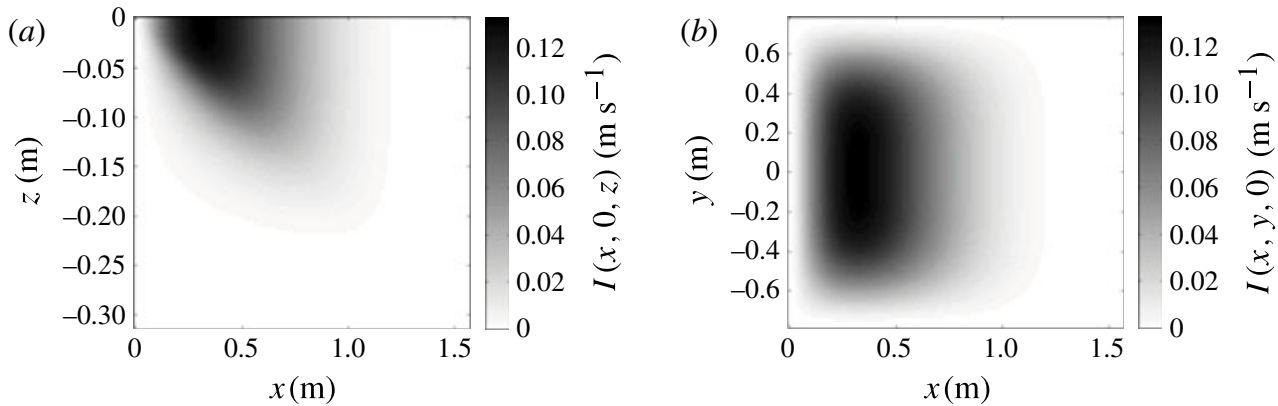

FIGURE 3. Impulse due to the body force parametrizing a breaking event. The plot on the left shows the impulse in the $(x, z)$ plane at $y=0$ while the plot on the right shows the impulse in the $(x, y)$ plane at $z=0$; based on forcing given in SMM2004, which is in the $\hat{\boldsymbol{x}}$ direction.

effects of the body force with a uniform surface stress of equivalent total momentum transfer, highlighting the marked impact of intermittent body forces, or breaking, on the dynamics of the water column. The impulse per unit mass, $\boldsymbol{I}$, imparted to a fluid by a body force per unit mass $\boldsymbol{F}$ is defined as

$$
\boldsymbol{I}=\int_{0}^{\mathscr{T}} \boldsymbol{F}(\boldsymbol{x}, t) \mathrm{d} t,
$$

where $\mathscr{T}$ is the duration of the forcing event (Lamb 1932, §119). Figure 3 shows the impulse of the body force, modelled after a deep water breaking wave, proposed by SMM2004. 
Sullivan, McWilliams \& Melville (2007, SMM2007) then extended the use of their model to include an ensemble of breaking events and explored the ramifications of these impulses on the underlying dynamics, finding that upper ocean processes respond strongly to the addition of wave breaking. Restrepo et al. (2011) used a qualitatively similar parametrization of the body force, as well as a scaling assumption that the velocity induced by the breaking is weak, to analytically solve for the behaviour of currents and waves in the presence of wave breaking. While the effects of breaking may be weak in the mean (Hasselmann 1974), the velocity due to breaking is $O(c)$ over short time scales (Rapp \& Melville 1990).

The ability to reproduce the vortical behaviour found in the laboratory (MVW) using an impulsive body force (SMM2004) implies that this is a viable model of wave breaking. The dynamics of impulsively forced fluids, and in particular the relationship between impulse and vorticity, is well known and in cases where the impulse has an axial symmetry, the resulting structures are known as vortex rings (see, for instance, Batchelor 1967, §§7.1 and 7.2; Saffman 1992).

The most striking feature of the vortex ring is its robust coherence (Shariff \& Leonard 1992). Theoretical investigations of vortex dynamics are complicated by the details of the vorticity distributions within their cores; however, for certain simple systems, closed-form equations describing integral scalar quantities of the vortex ring (i.e. impulse, energy, circulation) exist (Batchelor 1967, § 7.2). For instance, Helmholtz (1858) described, and Taylor (1953) quantified, a simple experiment to create a half vortex ring by forcing a partially submerged disc along its axis of symmetry in a fluid and then quickly removing it (see figure 5).

Theoretical descriptions of vorticity generation by breaking deep-water waves have been sparse, although descriptive models have been hypothesized. Csanady (1994) argued that breaking will generate vertical eddies at the surface, contributing to the Craik-Leibovich II (CL II) mechanism (Craik \& Leibovich 1976; Leibovich 1983) and enhancing Langmuir circulation, a suggestion supported by the numerical modelling of SMM2007. Similarly, Peregrine (1999) noted that Helmholtz's (1858) vorticity theorems imply that the vortex filament generated by a deep-water breaking wave is topologically equivalent to a half vortex ring (see figure 4). These studies, however, have stopped short of making quantitative predictions about the vorticity and circulation generated by breaking waves except in the case of shallow water, where the vorticity is bounded by the surface and the bottom (see, for instance, Peregrine 1998). The recent numerical efforts mentioned above have started to elucidate the importance of breaking in mixed-layer processes, but many of the basic theoretical mechanisms are still unclear (Thorpe 2005). In this paper we make progress in this direction by following SMM2004 and SMM2007 in assuming that the effects of a breaking wave can be modelled by an impulsively forced fluid, allowing us to find a relationship between the energy lost due to breaking and the circulation that is generated.

The organization of the paper is as follows. In $\S 2$ we introduce governing equations for a homogeneous incompressible fluid and derive from this an equation for the generated circulation. In $\S 3$, we associate the forcing with the effects due to a breaking wave and make scaling arguments supporting a relationship between the circulation and the characteristic variables of a breaking wave. Next, we compare the theoretical predictions with the available laboratory data. The results are discussed in $\$ 4$. 
FIgURE 4. Description, proposed by Peregrine (1999), of the topological shape (a half vortex ring) of the flow shortly after a breaking event. The surface of the torus denotes the vortex filament and the arrows indicate the direction of the velocity.

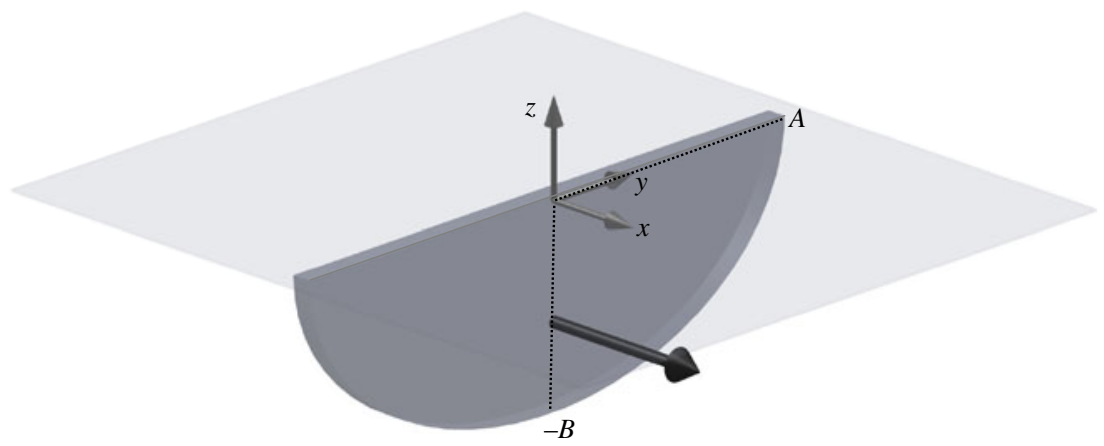

FIGURE 5. Sketch of a thin elliptical half disc of major axis length $2 A$ and minor axis length $2 B$ being forced from rest to a velocity $U \hat{\boldsymbol{x}}$ through a fluid with surface at $z=0$. Here $z<0$ corresponds to water while $z>0$ corresponds to air.

\section{The breaking circulation model}

\subsection{Governing equations}

Consider an incompressible homogeneous inviscid fluid with a free surface. The governing equations (i.e. Euler's equations) are

$$
\frac{\mathrm{D} \boldsymbol{u}}{\mathrm{D} t}=-\frac{1}{\rho} \nabla p+g \hat{z} ; \quad \nabla \cdot \boldsymbol{u}=0,
$$

where $\boldsymbol{u}$ is the fluid velocity, $\rho$ is the density of water, $g$ is the acceleration due to gravity and $p$ is the pressure. 
The present study is intended to characterize the post-breaking, ensemble-average, large-scale flow response to a breaking wave. To this end, we define the ensembleaveraging operator $\overline{()}$ such that

$$
\overline{\boldsymbol{G}(\boldsymbol{x}, t ; N)} \equiv \frac{1}{N} \sum_{n=1}^{N} \boldsymbol{G}(\boldsymbol{x}, t, n),
$$

where $\overline{\boldsymbol{G}}$ is the ensemble-average of the variable $\boldsymbol{G}(\boldsymbol{x}, t, n)$, measured during the $n$th realization of the experiment (where each realization has identical initial and boundary conditions), with total number of repeats $N$. For a thorough discussion of the properties of this averaging operator, see also Andrews \& McIntyre (1978, §2) and Kundu, Cohen \& Dowling (2012, § 12.3).

The measured fluctuating component of the variable, $\boldsymbol{G}^{\prime}(\boldsymbol{x}, t, n)$, is then defined as

$$
\boldsymbol{G}^{\prime}(\boldsymbol{x}, t, n)=\boldsymbol{G}(\boldsymbol{x}, t, n)-\overline{\boldsymbol{G}(\boldsymbol{x}, t ; N)} .
$$

The true ensemble-average and fluctuating components are just the limits of (2.2) and (2.3) as $N \rightarrow \infty$. The error associated with keeping $N$ finite can be estimated by conducting a number of repeat realizations of the experiment and then estimating the convergence of the measured quantity as a function of $N$ (see further discussion in $\S 3.2)$.

Now, we decompose the breaking-induced velocity $\boldsymbol{u}$ into mean, $\overline{\boldsymbol{u}}$, and fluctuating (i.e. turbulent), $\boldsymbol{u}^{\prime}$, components, with respect to this averaging operator, so that

$$
\boldsymbol{u}(\boldsymbol{x}, t, n)=\overline{\boldsymbol{u}(\boldsymbol{x}, t ; N)}+\boldsymbol{u}^{\prime}(\boldsymbol{x}, t, n),
$$

where, by definition, $\overline{\left(\boldsymbol{u}^{\prime}\right)}=0$. If we substitute this decomposition into (2.1), and then take the ensemble-average of the resulting equation, we find (see, for instance, Pope $2000, \S 4)$

$$
\frac{\partial \overline{u_{i}}}{\partial t}+\frac{\partial}{\partial x_{j}}\left(\overline{u_{i}} \overline{u_{j}}\right)+\frac{\partial}{\partial x_{j}} \overline{\left(u_{i}^{\prime} u_{j}^{\prime}\right)}=-\frac{1}{\rho} \frac{\partial \bar{p}}{\partial x_{i}}+g \hat{x}_{3},
$$

where the subscript $i(i=1,2,3)$ denotes the $i$ th Cartesian component of a vector (e.g. $x_{1}=x, x_{2}=y, x_{3}=z$ ), summation over repeated indices is assumed, and $\bar{p}$ represents the ensemble-average pressure. The influence of the turbulent velocity fluctuations on the mean flow is represented by the forcing associated with the Reynolds stress tensor $\overline{\left(u_{i}^{\prime} u_{j}^{\prime}\right)}$. We note that (2.5) is an inviscid version of the Reynoldsaveraged Navier-Stokes equations (see Pope 2000) with a free surface.

Now, instead of solving (2.5) directly, we will consider the circulation, $\Gamma$, of the ensemble-average velocity field. As will be shown below, the circulation is an integral scalar quantity governed by (an ensemble-average version of) Kelvin's circulation theorem and has established closed forms for vortex rings, which can be written solely in terms of the characteristic variables of a breaking wave.

Consider the circulation of the ensemble-average velocity field, defined as

$$
\Gamma \equiv \oint_{\bar{C}} \bar{u} \cdot \mathrm{d} \boldsymbol{\ell}
$$

where $\bar{C}$ is a closed material contour moving with velocity $\overline{\boldsymbol{u}}$, which will be further specified below. Next, the ensemble-average total derivative is defined as

$$
\frac{\overline{\mathrm{D}}}{\mathrm{D} t}=\frac{\partial}{\partial t}+\overline{\boldsymbol{u}} \cdot \nabla
$$


so that the evolution of (2.6) is then given by

$$
\frac{\overline{\mathrm{D}} \Gamma}{\mathrm{D} t}=\oint_{\bar{C}} \frac{\overline{\mathrm{D}} \overline{\boldsymbol{u}}}{\mathrm{D} t} \cdot \mathrm{d} \boldsymbol{\ell}+\oint_{\bar{C}} \overline{\boldsymbol{u}} \cdot \frac{\overline{\mathrm{D}}}{\mathrm{D} t} \mathrm{~d} \boldsymbol{\ell} .
$$

The second term on the right-hand side of (2.8) can we rewritten as (Batchelor 1967, $\S \S 3.1$ and 5.2)

$$
\oint_{\bar{C}} \overline{\boldsymbol{u}} \cdot \frac{\overline{\mathrm{D}}}{\mathrm{D} t} \mathrm{~d} \boldsymbol{\ell}=\oint_{\bar{C}} \overline{\boldsymbol{u}} \cdot(\mathrm{d} \boldsymbol{\ell} \cdot \nabla \overline{\boldsymbol{u}})=\oint_{\bar{C}} \nabla\left(\frac{\overline{\boldsymbol{u}} \cdot \overline{\boldsymbol{u}}}{2}\right) \cdot \mathrm{d} \boldsymbol{\ell},
$$

which, by application of Stokes' theorem and the fact that the curl of the gradient of a scalar function vanishes, is identically zero.

Next, we substitute (2.5) into the first term on the right-hand side of (2.8), so that we find

$$
\oint_{\bar{C}} \frac{\overline{\mathrm{D}} \overline{\boldsymbol{u}}}{\mathrm{D} t} \cdot \mathrm{d} \boldsymbol{\ell}=\oint_{\bar{C}}\left(-\frac{\partial}{\partial x_{j}} \overline{\left(u_{i}^{\prime} u_{j}^{\prime}\right)}-\frac{1}{\rho} \frac{\partial \bar{p}}{\partial x_{i}}+\frac{\partial\left(g x_{3}\right)}{\partial x_{i}}\right) \mathrm{d} \ell_{i} .
$$

First, we note that the second and third terms on the right-hand side of (2.10), involving the ensemble-average pressure $\bar{p}$ and the acceleration due to gravity $g$, will be zero because, again by Stokes' theorem, the argument of these integrals is the curl of the gradient of a scalar function.

Next, we perform the canonical decomposition of the Reynolds stress tensor into isotropic and deviatoric anisotropic components (see, for instance, Pope 2000, §4.2):

$$
\overline{\left(u_{i}^{\prime} u_{j}^{\prime}\right)}=\left(\Pi \delta_{i j}+\mathscr{D}_{i j}\right),
$$

where

$$
\Pi=\frac{1}{3} \overline{\left(u_{i}^{\prime} u_{i}^{\prime}\right)}=\frac{2}{3} e(\boldsymbol{x}, t),
$$

$\Pi \delta_{i j}$ (with $\delta_{i j}=1$ for $i=j$ and 0 otherwise) is the isotropic (normal) stress, $\Pi$ is a scalar function proportional to the turbulent kinetic energy $e(\boldsymbol{x}, t) \equiv \overline{\left(\boldsymbol{u}^{\prime} \cdot \boldsymbol{u}^{\prime}\right)} / 2$ and $\mathscr{D}_{i j}$ is the anisotropic (shear) stress, given by

$$
\mathscr{D}_{i j}=\overline{\left(u_{i}^{\prime} u_{j}^{\prime}\right)}-\frac{2}{3} e(\boldsymbol{x}, t) \delta_{i j} .
$$

Therefore, the term related to the Reynolds stresses in the governing equation for the circulation, i.e. (2.10), is equivalent to

$$
\oint_{\bar{C}}-\frac{\partial}{\partial x_{j}} \overline{\left(u_{i}^{\prime} u_{j}^{\prime}\right)} \mathrm{d} \ell_{i}=\oint_{\bar{C}}-\frac{\partial}{\partial x_{j}}\left(\Pi \delta_{i j}+\mathscr{D}_{i j}\right) \mathrm{d} \ell_{i} .
$$

The contour integral of the normal component of the Reynolds stress tensor, $\nabla \Pi$, will be zero, which again can be seen by application of Stokes' theorem and noting that the curl of the gradient of a scalar function vanishes, so that (2.14) reduces to

$$
\oint_{\bar{C}}-\frac{\partial}{\partial x_{j}} \overline{\left(u_{i}^{\prime} u_{j}^{\prime}\right)} \mathrm{d} \ell_{i}=\oint_{\bar{C}}-\frac{\partial \mathscr{D}_{i j}}{\partial x_{j}} \mathrm{~d} \ell_{i} .
$$

Now, the laboratory studies under consideration in this paper use dispersive focusing of compact unidirectional wave groups to induce unsteady quasi-two-dimensional breaking waves. For times much less and much greater than the time at which breaking occurs, the wave groups are accurately described by linear theory, which predicts that the momentum transported by these wave packets is solely in the 
direction of wave propagation (see, for instance, Phillips 1977, §3), which throughout this work is along the $x$-axis.

That is, if $\mathcal{M}^{o}\left(\boldsymbol{x}, t \ll t_{b}\right)=\mathcal{M}^{o}\left(\boldsymbol{x}, t \ll t_{b}\right) \hat{\boldsymbol{x}}$, where $t_{b}$ represents the time at which the breaking event occurs, is the momentum in the wave field before breaking, and $\mathcal{M}^{f}\left(\boldsymbol{x}, t \gg t_{b}\right)=\mathcal{M}^{f}\left(\boldsymbol{x}, t \gg t_{b}\right) \hat{\boldsymbol{x}}$ is the momentum in the wave field after breaking, the difference $\Delta \mathcal{M}=\left(\mathcal{M}^{o}-\mathcal{M}^{f}\right) \hat{\boldsymbol{x}}$ is transferred to the water column by the breaking process. Note, as mentioned in the introduction, some of the momentum lost from the wave field due to breaking will go into other phenomena besides current generation. See $\S 4$ for further discussion (see also SMM2004, §4; SMM2007, §3). If the breaking event occurs for a time $\mathscr{T}$, then we see that $\Delta \mathcal{M} / \mathscr{T}$ represents the average force exerted by the breaking wave on the water column during the breaking event. Now, the details of the momentum loss from the wave field are unknown during the highly nonlinear breaking process, where locally, vertical exchanges of momentum can occur. However, by the above arguments, the average forcing corresponding to the change in the wave packet's momentum is entirely in the direction of wave propagation.

Shortly after the breaking event, the stresses due to quasi-two-dimensional breaking waves have been reported by, for instance, Rapp (1986), MVW and Drazen \& Melville (2009). However, during the breaking process the exact form of the Reynolds stress tensor is unknown, so that we must choose how to close these equations. Based on the above discussion of the momentum lost by the wave field, we will assume that the mean flow induced during the breaking event is solely in the direction of wave propagation, so that the forcing, associated with the shear Reynolds stresses of the breaking induced turbulent velocities, also acts along the $x$-axis.

Therefore, we parametrize the forcing terms, related to the shear Reynolds stresses in our governing equation for the mean flow, i.e. (2.15), as

$$
\oint_{\bar{C}}-\frac{\partial \mathscr{D}_{i j}}{\partial x_{j}} \mathrm{~d} \ell_{i} \equiv \oint_{\bar{C}} \bar{F} \delta_{i 1} \mathrm{~d} \ell_{i},
$$

where the anisotropic stress term is related to the body force $\overline{\boldsymbol{F}}$, with $\overline{\boldsymbol{F}}=\overline{\boldsymbol{F}} \hat{\boldsymbol{x}}$.

Therefore, the governing equation for the circulation reduces to

$$
\frac{\overline{\mathrm{D}} \Gamma}{\mathrm{D} t}=\frac{\overline{\mathrm{D}}}{\mathrm{D} t} \oint_{\bar{C}} \overline{\boldsymbol{u}} \cdot \mathrm{d} \boldsymbol{\ell}=\oint_{\bar{C}} \overline{\boldsymbol{F}} \cdot \mathrm{d} \boldsymbol{\ell},
$$

which is an inhomogeneous form of Kelvin's circulation theorem, describing the evolution of the circulation of the ensemble-average flow generated by the body force $\overline{\boldsymbol{F}}$, where the body force will model the bulk scale effects of a breaking wave on the water column. A body force acting on a fluid with a free surface can lead to the generation of both waves and vortices (see, for instance, Bühler 2007). Post-breaking flow is analysed in the laboratory experiments of Rapp \& Melville (1990) and MVW, where wave breaking is due to compact wave groups, which focus, break and then propagate away. Based on this we can assume that after the breaking event, in the compact region where the forcing occurred and beyond, the free surface effectively acts like a rigid lid (see also SMM2004).

To accurately model the long time evolution of the flow one must also include effects due to viscosity. However, we note that the laboratory experiments of MVW (see their figure 14) show that circulation decreases by less than an order of magnitude for a time interval of more than 50 wave periods after breaking. 


\subsection{Vortex generation}

The exact functional form of the ensemble-average body force $\overline{\boldsymbol{F}}$ cannot be resolved, but following SMM2004, can be guided by laboratory data. We assume that the body force is compact in space and time, and is symmetric, about $y=0$, along the crest of the breaking wave (see figure 3). It is natural to then consider the simple model proposed by Helmholtz (1858, see also Tait 1867), and quantified by Taylor (1953) (see also Saffman 1992, § 6.4), in which a half vortex ring is generated by impulsively forcing a thin disc, half submerged, through a perfect fluid with no flow separation. Breaking waves may have an asymmetry between the horizontal and vertical scales, so that a generalization of Taylor's (1953) work to elliptical vortex rings, by Dhanak \& De Bernardinis (1981), is more applicable to our study. Elliptical vortex rings can be generated by slightly modifying the thought experiment of Helmholtz (1858) to consider the flow generated by impulsively forcing a thin elliptical disc, submerged along its major axis to the depth of its semi-minor axis, through a fluid.

More precisely, we assume the forcing, $\overline{\boldsymbol{F}}$, acts like a thin elliptical disc submerged along its major axis, of length $2 A$, to the depth of its semi-minor axis, of length $B$, being impulsively forced from rest to a speed $U$ in a perfect fluid (see figure 5) with no flow separation. This problem was considered by Dhanak \& De Bernardinis (1981) in their work on elliptical vortex rings.

Now, Dhanak \& De Bernardinis (1981) find that the velocity potential at the surface of the disc is

$$
\phi\left(x=0^{ \pm}, y, z\right)=\mp \frac{U b}{\mathscr{E}(e)} \sqrt{1-\frac{y^{2}}{A^{2}}-\frac{z^{2}}{B^{2}}},
$$

where \pm refers to the front and rear of the disc, respectively; $e$ is the eccentricity and $\mathscr{E}(e)$ is the complete elliptic integral of the second kind:

$$
\mathscr{E}(e)=\int_{0}^{\pi / 2} \sqrt{1-e^{2} \sin ^{2} \theta} \mathrm{d} \theta ; \quad e^{2} \equiv \frac{A^{2}-B^{2}}{A^{2}} .
$$

The circulation around a contour starting at $(x, y, z)=\left(0^{+}, 0,0\right)$ and ending at $\left(0^{-}, 0,0\right)$ is given by Dhanak \& De Bernardinis (1981)

$$
\Gamma=\phi\left(0^{-}, 0,0\right)-\phi\left(0^{+}, 0,0\right)=\frac{2 U B}{\mathscr{E}(e)} .
$$

Following Taylor (1953), we assume the disc is suddenly dissolved, so that the induced motion may now be described as being due to a collection of elliptic vortex lines, with axis ratios the same as that of the disc, over the plane of the disc. From (2.18) it is clear that the velocity is strongest at the edge of the disc, implying that the flow will tend to roll up around this region (see also Saffman 1992, §§ 6.3, 8.4), forming an elliptical vortex ring.

We assume the rolling up of the vortex sheet will not change the circulation around the core (Taylor 1953; Saffman 1992) so that the circulation of the generated vortex ring will be the same as the circulation of the forced disc, as given in (2.20). Dhanak \& De Bernardinis (1981) found that elliptical vortex rings will oscillate in shape as they propagate, and for cases where the vortex ring has large eccentricity, can potentially break-up into multiple rings. The time scale of these oscillations is on the order of $4 \pi A^{2} / \Gamma$. For the laboratory experiments considered in this paper, $4 \pi / \Gamma$ is of the order of $10^{2} \mathrm{~m}^{-2} \mathrm{~s}$ (see $\S 3$ ) while $A$ is taken to be large, so that the time 
scale of these oscillations is much larger than the times in which we are interested in describing the circulation.

\section{Circulation of breaking waves}

Vortex lines must start and end on the boundaries, or be closed loops within the fluid. This implies that for three-dimensional deep-water breaking waves the distribution of vorticity must be topologically equivalent to a half vortex ring, since the vortex lines induced by the event will not reach the bottom (Peregrine 1999; Csanady 1994; Thorpe 2005, see also figure 4). Note that in two dimensions the vorticity induced by breaking takes a form such that the kinematics can be described by those of a point vortex, which is a limiting case of the three-dimensional vortex ring as $A \rightarrow \infty$ with $B$ finite (see figure 2; see also Rapp \& Melville 1990; MVW). Along with the considerations of the structure of the impulse due to breaking discussed in $\S 2.2$, we now see that there is a direct relationship between the flow response to an impulsively forced thin disc moving through a fluid and that due to a breaking wave. Therefore, we are in a position to apply the relationship established in (2.20), between the circulation and the characteristic variables of the forced disc, to the problem of wave breaking.

Now, from the laboratory experiments of Rapp \& Melville (1990), and the scaling arguments of Drazen et al. (2008), we expect the variables parametrizing the forced disc to be related to those describing a breaking wave; but precisely how is not immediately clear. In order to make progress, we turn to scaling arguments.

\subsection{Scaling of circulation}

We present two separate scaling arguments to quantify, to within a constant, the circulation induced by a breaking wave. First, we will make a scaling argument based on dynamical considerations relating the momentum lost from the wave field due to breaking to the variables describing the generated vortex. Second, we make scaling arguments based on the geometry and kinematics of both a plunging and spilling breaking wave.

\subsubsection{Scaling based on dynamical considerations}

A fully three-dimensional breaking wave will start to break at a point in space and subsequently this breaking spreads along the length of the crest of the breaking wave, with associated time scale $\tau$. We assume that this occurs on a much faster time scale than the wave period, $T$, of the breaking wave (i.e. $\tau \ll T$ ), so that following SMM2004, the crest length is taken to be constant. Finally, we assume the initial depth of penetration of a breaking wave is generally much smaller than the resulting length scale of the crest; that is, $A \gg B$, so that $\mathscr{E}(e) \approx 1$ in $(2.20)$ :

$$
\Gamma \approx 2 U B .
$$

Note, as mentioned above, that we are assuming the circulation is conserved during the roll-up process, so that (3.1) is also the circulation of the vortex generated by the impulsive event.

The fluid impulse $\mathscr{P}$ (Batchelor 1967, §7.2), associated with the flow of the forced elliptical disc presented in $\S 2.2$ is given by Dhanak \& De Bernardinis (1981)

$$
\mathscr{P}=\rho \frac{2 \pi A B^{2} U}{3 \mathscr{E}(e)},
$$


where the variables are as defined in that section (see also figure 5). Accounting for the above assumption that the major axis of the disc is much larger than the minor axis, equation (3.2) reduces to

$$
\mathscr{P}=\rho \frac{2 \pi A B^{2} U}{3} .
$$

Comparing this with (3.1), we see that

$$
\Gamma=\frac{1}{\rho} \frac{3 \mathscr{P}}{2 \pi A B} .
$$

By considering the reduction of energy and momentum densities of the wave field due to breaking, the total energy lost by a breaking event per unit length of breaking crest is given by (Phillips 1985; see also SMM2007, §3.1)

$$
E_{l}=c M_{l},
$$

where $M_{l}$ is the total momentum loss per unit length of breaking crest and $c$ is the phase speed of the breaking wave. Here $E_{l}$ is related to the energy dissipation rate per unit length of breaking crest $\epsilon_{l}$ by

$$
E_{l}=\int_{0}^{\mathscr{T}} \epsilon_{l} \mathrm{~d} t,
$$

where $\mathscr{T}$ is a constant of $O(T)$ (Rapp \& Melville 1990; see also SMM2004, SMM2007), $T$ is the period of the breaking wave, and the energy dissipation rate $\epsilon_{l}$ is given by (1.1) and (1.3), that is (Drazen et al. 2008)

$$
\epsilon_{l}=\beta \frac{(h k)^{5 / 2} \rho c^{5}}{g} .
$$

Now, the loss of momentum from the wave field will go into the generation of the rotational flow under consideration (Rapp \& Melville 1990, §5.4; see also § 2.1 and $\S 4$ here for further discussion on the momentum lost by the wave field), which implies that $M_{l}$ can be related to the impulse of the forced disc $\mathscr{P}$ :

$$
\mathscr{P}=\int M_{l} \mathrm{~d} y=\iint_{0}^{\mathscr{T}} \frac{\epsilon_{l}}{c} \mathrm{~d} t \mathrm{~d} y,
$$

where the last equality comes from (3.5), (3.6) and (3.7), and the spatial integrals are over the entire region where the forcing acts.

Based on Rapp \& Melville (1990), we assume that the semi-minor axis of the disc $B$ scales with the depth of the penetration of the fluid, and hence the height of the wave at breaking $h$. Also, we assume that the major axis of the elliptical disc $2 A$ scales with the crest length of the breaking wave.

Therefore, substituting (3.7) into (3.8) and equating with (3.4), we find

$$
\Gamma \sim(h k)^{3 / 2} c \lambda \sim(h k)^{3 / 2} \frac{c^{3}}{g},
$$

where the linear dispersion relation was used to rewrite the wavelength in terms of the phase speed and the gravitational constant. From (3.9) we conclude that

$$
\Gamma=\varpi \frac{(h k)^{3 / 2} c^{3}}{g},
$$


where $\varpi$ is a constant. Recall that the forced disc corresponds to the ensemble-average body force model $\overline{\boldsymbol{F}}$, so that $\Gamma$ is the generated circulation corresponding to the ensemble-averaged velocity field $\overline{\boldsymbol{u}}$, i.e. (2.6). Therefore, to within a scaling constant, we have described this circulation in terms of the characteristic variables of the breaking wave.

\subsubsection{Plunging breaking waves}

Next, we present scaling arguments for the generation of circulation by both plunging and spilling quasi-two-dimensional breakers, using models based on the geometry and kinematics of breaking. This follows the work of Melville (1994) and especially Drazen et al. (2008), who hypothesized and tested a functional form of the breaking parameter $b,(1.3)$, based on an inertial model for plunging breakers. Romero et al. (2012) then showed that by introducing a threshold slope for breaking, these results held over the available laboratory data extending from the onset of weak breaking to very strong plunging breaking waves (see figure 1).

We assume that the plunging breaker has amplitude $a$ and height at breaking $h$ (see figure $6 a$ ), the toe of the breaking wave follows a ballistic trajectory once it begins to form and has velocity $w=\sqrt{2 g h}$ at impact with the water (Drazen et al. 2008). Before breaking, the flow is irrotational, with vorticity being introduced when the toe of the breaker reconnects with the surface (Hornung, Willert \& Turner 1995).

Inertial scaling of the vorticity just after the breaking event implies $\omega=\zeta \tilde{u} / \tilde{l}$ where $\omega$ is the magnitude of vorticity, $\tilde{u}$ and $\tilde{l}$ are the characteristic velocity and length scales of the flow, respectively, and $\zeta$ is a constant parameter of order unity. Drazen et al. (2008) assumed the velocity scale is set by $w=\sqrt{2 g h}$, the length scale by $h$ and the area of the cloud of turbulence induced by the breaking, $\mathscr{A}$, is $\pi h^{2} / 4$ (see figures $6 a$ and $6 b$; see also figure 3 in Drazen et al. 2008).

Now, we are interested in finding the circulation of the ensemble-average velocity field, which we recall is given by (2.6):

$$
\Gamma=\oint_{\bar{C}} \bar{u} \cdot \mathrm{d} \ell=\int_{\overline{\mathscr{A}}} \overline{\boldsymbol{\omega}} \cdot \mathrm{d} \mathcal{A}
$$

where the second equality is from Stokes' theorem, $\overline{\boldsymbol{\omega}} \equiv \nabla \times \overline{\boldsymbol{u}}$ and $\overline{\mathscr{A}}$ is the area bounded by $\bar{C}$.

Recall that Rapp \& Melville (1990, § 5) found that the mean and turbulent velocities induced by wave breaking had comparable magnitudes, so that we assume the generated ensemble-average velocity is of the same order as the integral velocity scale, $w$, used by Drazen et al. (2008). Furthermore, Rapp \& Melville (1990, §4.3) found that the deviation in the area of mixing of the broken fluid, for an individual realization of a breaking event, was within an order one constant of the ensembleaverage value. This implies that the mean area of entrainment induced by the breaking event $\overline{\mathscr{A}}$ is of the same order as the turbulent cloud used by Drazen et al. (2008), $\mathscr{A}$. Therefore, equation (3.11) becomes

$$
\Gamma=\int_{\overline{\mathscr{A}}} \overline{\boldsymbol{\omega}} \cdot \mathrm{d} \mathcal{A} \sim \int_{\mathscr{A}} \omega \mathrm{d} \mathscr{A}=\zeta \frac{\pi}{4} h \sqrt{2 g h},
$$

so that, using the linear dispersion relation, we find

$$
\Gamma=\alpha \frac{(h k)^{3 / 2} c^{3}}{g}
$$


(a)

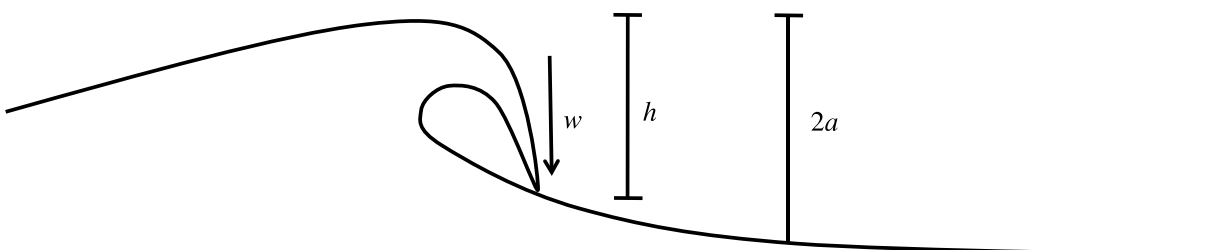

(b)

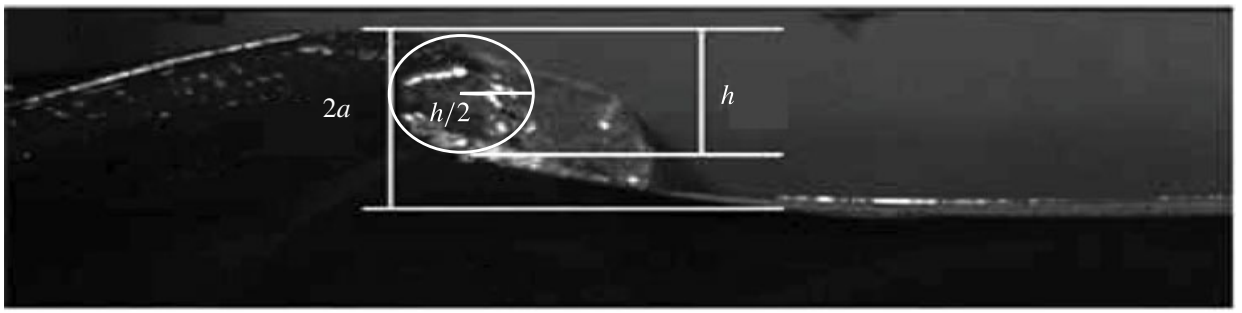

(c)

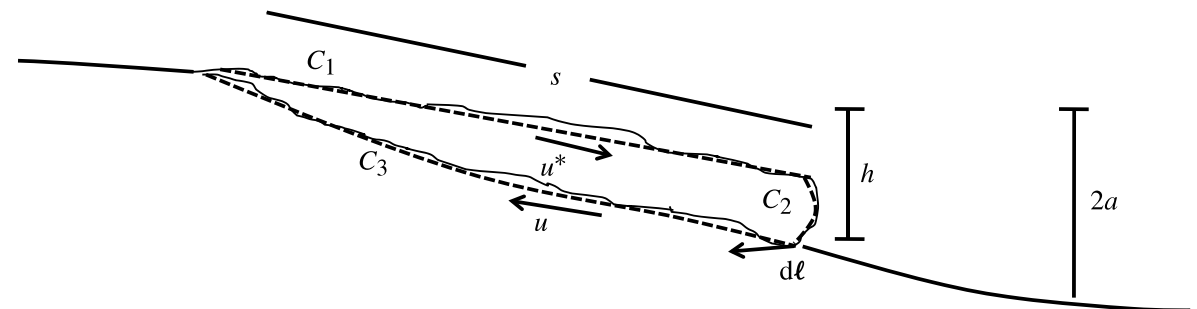

FIGURE 6. Schematic of $(a)$ the geometry of a plunging breaker, following Drazen et al. (2008) where $w$ is the velocity of the toe of the breaker as it penetrates the water and $(b)$ a video frame of a plunging breaking wave as the toe connects with the surface, adapted from Drazen et al. (2008), where we see the turbulence cloud corresponding to the vorticity induced by the breaking, i.e. the white circle, has the approximate cross-section $\pi h^{2} / 4$. $(c)$ A spilling breaker based on Longuet-Higgins \& Turner (1974): $u^{*}$ and $u$ denote the velocity of the whitecap and underlying motion due to the wave, respectively, in a reference frame moving at the phase speed of the breaking wave and $s$ is the downslope distance as measured from the crest of the wave. The dashed lines represents the contour of integration $C$ with tangent vector $\mathrm{d} \ell$. See the text for details. In $(a)-(c), a$ and $h$ represent the amplitude and height of the breaking wave.

where $\alpha$ is a constant. This result, up to the scaling constant, is in accordance with (3.10).

\subsubsection{Spilling breaking waves}

Spilling breakers appear to be markedly different from their plunging counterparts; nevertheless, an analogous scaling model can be constructed to find a functional form for the circulation. Longuet-Higgins \& Turner (1974) modelled a spilling breaker as a steady turbulent gravity current riding down the forward fixed slope of the underlying breaking wave, and assumed the flow remained similar as it developed in time. Although this theory is formulated for steady motion, it was shown to approximately describe both the acceleration of the front of an unsteady spilling breaker, as well as the geometry of the breaking region (Longuet-Higgins \& Turner 1974, §§ 6, 7; see also Duncan 2001, §5.1), prompting its use as a possible model describing the geometry and kinematics of an unsteady spilling breaking wave. 
Longuet-Higgins \& Turner (1974) proposed that the speed along the slope, in a reference frame moving with the phase speed of the breaking wave, is $u^{*}=U^{*} \sqrt{g s^{\prime}}$ and $u=U \sqrt{g s^{\prime}}$ for the whitecap and underlying fluid, respectively. Here $U^{*}$ and $U$ are constants and $s^{\prime} \in(0, s)$ represents the distance downslope from the crest of the wave, where $s$ is proportional to $h$, the height of the breaking wave (see figure $6 c$ ).

Now, the scaling of the ensemble-average velocity, and area of entrainment of the broken fluid, discussed in the section on plunging breaking waves (§3.1.2) were also observed by Rapp \& Melville (1990) to be true for spilling breaking waves. Therefore, we assume that the mean induced velocity $\overline{\boldsymbol{u}}$ scales with the total induced velocity $\boldsymbol{u}$ and that the mean contour $\bar{C}$ scales with $C$, so that

$$
\Gamma=\oint_{\bar{C}} \bar{u} \cdot \mathrm{d} \boldsymbol{\ell} \sim \oint_{C} \boldsymbol{u} \cdot \mathrm{d} \boldsymbol{\ell} .
$$

Assuming the whitecap remains thin (Longuet-Higgins \& Turner 1974) and the velocity along the front remains finite, the circulation generated along $C_{2}$ will be small compared with the contributions along $C_{1}$ and $C_{3}$, so that the circulation around the contour, $C=C_{1}+C_{2}+C_{3}$, is approximated by

$$
\begin{aligned}
\Gamma & =\oint_{\bar{C}} \overline{\boldsymbol{u}} \cdot \mathrm{d} \boldsymbol{\ell} \sim \oint_{C} \boldsymbol{u} \cdot \mathrm{d} \boldsymbol{\ell} \approx \int_{C_{1}} \boldsymbol{u} \cdot \mathrm{d} \boldsymbol{\ell}+\int_{C_{3}} \boldsymbol{u} \cdot \mathrm{d} \boldsymbol{\ell} \\
& =\int_{0}^{s} U^{*} \sqrt{g s^{\prime}} \mathrm{d} s^{\prime}+\int_{s}^{0} U \sqrt{g s^{\prime}} \mathrm{d} s^{\prime}=\frac{2}{3}\left(U^{*}-U\right) \sqrt{g s^{3}},
\end{aligned}
$$

to give

$$
\Gamma=\varkappa \frac{(h k)^{3 / 2} c^{3}}{g},
$$

where we have again used the linear dispersion relationship and $x$ represents a constant of proportionality. We note that a similar argument, based on this model, gives the same functional form of the energy dissipation rate found by Drazen et al. (2008) for plunging breaking waves, that is, (3.7).

As was discussed in $\S 2$, we assume the circulation imparted by the breaking will be conserved, so that the above relationships will describe the circulation of the postbreaking flow. Therefore, we conclude that (3.10), (3.13) and (3.17) are all equivalent, up to a scaling constant, and characterize the generated circulation, corresponding to the ensemble-average velocity, for both spilling and plunging breakers, based on the characteristic variables of the breaking wave.

It should be noted that a simpler dimensional analysis argument, in the spirit of Drazen et al. (2008), can give us this same functional form for the circulation by assuming that it is a function of only gravity and the height of the breaking wave; namely, $\Gamma=\Gamma(g, h)$.

Through the wave slope at breaking $h k$, we can find a connection between the wave energy dissipation and the circulation. To this end, equating (3.10), (3.13) or (3.17) with (3.7), we have

$$
\Gamma=\frac{\Xi}{g}\left(\frac{g \epsilon_{l}}{\rho}\right)^{3 / 5},
$$




$\begin{array}{lccccc}\text { Experiment } & S & \text { Description } & f_{c}(\mathrm{~Hz}) & \begin{array}{c}\text { Number of } \\ \text { repeats }\end{array} & \begin{array}{c}\text { Time after } \\ \text { breaking }\end{array} \\ \text { RM } & 0.278 & \text { Spilling } & 0.88 & 10 & 4 \\ \text { RM } & 0.352 & \text { Plunging } & 0.88 & 10 & 4 \\ \text { MVW } & 0.320 & \text { Plunging } & 0.99 & 15 & 3 \\ \text { DM } & 0.360 & \text { Plunging } & 1.08 & 20 & 3.42 \\ \text { Rapp } & 0.420 & \text { Plunging } & 1.28 & 10 & 4\end{array}$

TABLE 1. Summary of relevant laboratory experiments and wave parameters for the circulation generated by quasi-two-dimensional breaking waves, with quantities as given in the original papers, used to test the model for circulation generated by breaking, i.e. (3.19). Here $S$ is as defined in (1.2) while $f_{c}$ represents the centre frequency of the input wave packet. RM, DM and Rapp correspond to Rapp \& Melville (1990), Drazen \& Melville (2009) and Rapp (1986), respectively, and all other names are as denoted in the text. Note that the available data includes both plunging and spilling breaking waves.

where $\Xi$ is a constant of proportionality. This simple relationship establishes a connection between the wave energy dissipated by a breaking event and the resulting circulation.

\subsection{Comparison with laboratory studies}

We now compare our model with the limited available laboratory data. The main features of the laboratory experiments, consistent with this study, are shown in table 1 and we refer the reader to the original papers for full details on the experimental techniques and laboratory configurations. The accessible laboratory experiments were conducted for quasi-two-dimensional breaking waves using a dispersive focusing technique, with breaking strength ranging from spilling to plunging. The studies under consideration also employed a technique to obtain spatial measurements of the induced velocity field, thus allowing for the calculation of the circulation generated by a breaking wave. For each experiment, an ensemble of runs (between 10 and 20 depending on the study) were conducted for a fixed set of initial wave parameters.

We consider the circulation of the vortex generated by the breaking wave, corresponding to the ensemble-averaged velocity field, at the earliest measured time after the breaking event (cf. equation (2.17)). Also, in order to obtain a consistent comparison amongst the different experiments, we sought to calculate the circulation at a common time after the breaking event. This occurred for times in the interval $\tilde{t} \in[3,4]$, where $\tilde{t} \equiv t / T_{c}$ ( $T_{c}$ is the centre period of the input wave group), after the breaking event, with the specific time dependent on the available data from the experiment in question (see table 1). Recall that Rapp \& Melville (1990) found that four wave periods after the breaking event, more than $90 \%$ of the energy lost by the wave field had been dissipated, while most of the momentum lost by the wave field was in the breaking generated mean flow.

Rapp \& Melville (1990) and Rapp (1986) used a laser doppler anemometer to measure the velocity field of the flow induced by breaking. The three cases from these experiments include both spilling and plunging breaking waves (see table 1). Each breaking scenario was repeated 10 times (the authors reported negligible differences between the mean velocity for 10 repeats versus 40 repeats). The original data from these laboratory experiments is unavailable, so that a description of the circulation was inferred from the published figures depicting the ensemble average velocity field 
induced by breaking (specifically figures 43b and 45b in Rapp \& Melville (1990) and figure 5.3.6b in Rapp (1986)). In particular, the average velocity along each segment of a rectangular contour, encompassing the vortex in question, was computed, from which the circulation was estimated.

Next, the laboratory experiments of MVW (see also White 1996) used digital particle image velocimetry (DPIV) to measure the velocity field induced by a plunging breaking wave. The authors found that the mean square velocity rapidly converged over an ensemble of events (see MVW, figure 4), so that the mean square velocity of a 15 member ensemble had a relative error of approximately $2 \%$ compared with a total sample size of 24 repeats. The authors then computed the circulation, corresponding to the ensemble average of 15 repeats, of the induced vortex three wave periods after the breaking event (see MVW, figure 5).

Finally, Drazen \& Melville (2009) used a similar DPIV technique to analyse properties of the flow induced by a plunging breaking wave. Drazen (personal communication) has provided us with archived data of 20 repeats of the velocity field induced by breaking from the study of Drazen \& Melville (2009, figure 2). Following the analysis of MVW, for each realization we compute the circulation around a closed rectangular contour that encompasses the vortex under consideration. We find that the circulation (non-dimensionalized by $g / c^{3}$ ) corresponding to the ensemble-averaged velocity field is 0.152 , with a standard deviation of 0.022 , which also corroborates the assumptions made to derive (3.13). This is shown in the error bar in figure 7.

Now, (3.10) and (3.13) or (3.17) imply that the (non-dimensional) circulation is

$$
\Gamma \frac{g}{c^{3}}=\mathscr{C}(h k)^{3 / 2}
$$

where $\mathscr{C}$ is a constant. Based on the laboratory results of Banner \& Peirson (2007, Appendix B; see also Rapp \& Melville 1990) and the analysis of Drazen et al. (2008, $\S 5$ ), we assume that the characteristic speed of the breaking wave is given by the phase speed of the centre frequency of the input wave packet. Next, as mentioned in the introduction, and following Drazen et al. (2008), instead of using the slope at breaking $h k$, which depends on nonlinear processes leading up to breaking, we consider the maximum predicted linear slope at breaking $S$, since this parameter is known a priori. Following Romero et al. (2012), a slope threshold and scaling factor for $S$ were introduced and obtained by a least-squares fit with the data, giving

$$
\Gamma \frac{g}{c^{3}}=0.85(S-0.058)^{3 / 2} \text {. }
$$

Figure 7 shows a comparison between the model described in (3.20) with the data given in table 1. Although only a limited amount of data is available, the relative agreement with the model is encouraging.

\section{Discussion}

We have proposed a simple relationship for the ensemble-averaged circulation generated by breaking deep-water surface gravity waves as a function of the characteristic variables of the breaking wave. Through scaling arguments, we have also clarified why the form of the governing equation for energy dissipation rate, as well as the generated circulation, should hold over the entire range of breaking, that is for both plunging and spilling breaking waves.

Note that Romero et al. (2012, equation (24); see also figure 1) found, via a visual fit, that the parameters that best fit, with respect to the available laboratory data for 


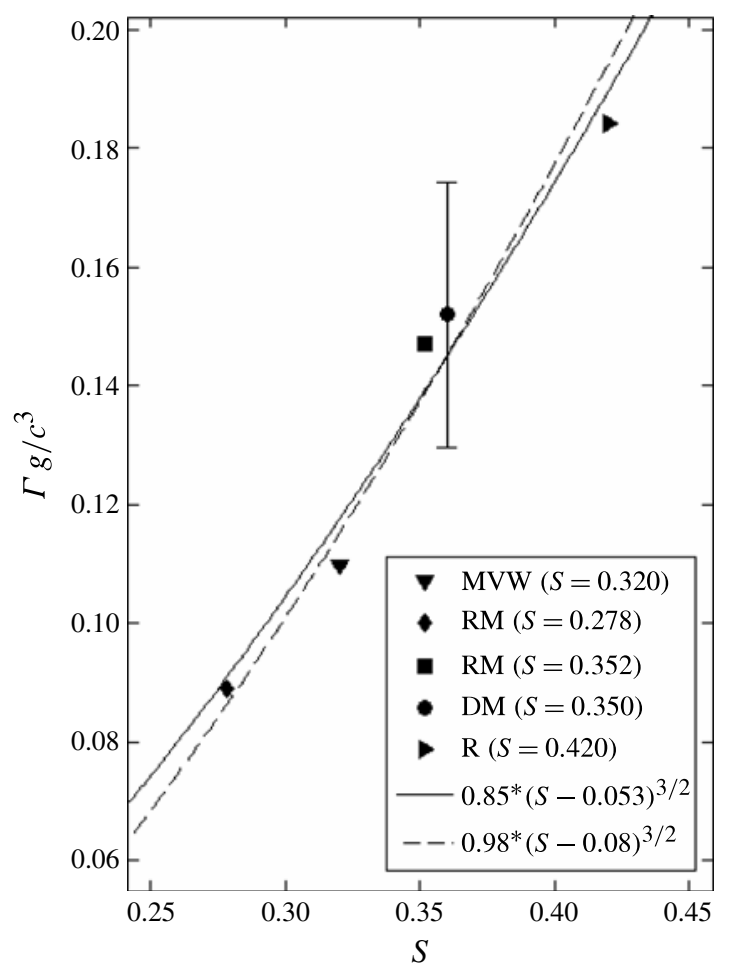

FIGURE 7. Comparison of normalized circulation versus maximum linear slope at breaking $S$ for the available laboratory data, which includes both spilling and plunging breaking waves. The sources of data in the legend are explained in table 1. The error bar on the DM data denotes the standard deviation of individual realizations versus the 20 member ensemble average. The solid line is given by (3.20) while the dashed line is based on the breaking strength parameter $b$, from Romero et al. (2012), and is given by (4.2). See the text for further details.

the energy dissipation rate per unit length of breaking crest, the model of Drazen et al. (2008) were $b(S)=0.4(S-0.08)^{5 / 2}$. Substituting this relation into (3.7), and subsequently (3.18), gives

$$
\Gamma \frac{g}{c^{3}}=0.58 \Xi(S-0.08)^{3 / 2} .
$$

A least-squares fit of $\Xi$ with the laboratory data gives $\Xi \approx 1.64$, so that

$$
\Gamma \frac{g}{c^{3}}=0.98(S-0.08)^{3 / 2},
$$

which is displayed by the dashed line in figure 7. Note that the scaling factor $\Xi$ is of order unity, and not inconsistent with the $O(1)$ scaling of constants in (3.10), (3.13) and (3.17).

It should also be noted that energy and momentum lost from the wave field to the water column due to breaking will go into other phenomena besides that which explicitly creates the large-scale flow that is analysed in this paper. Principal amongst these is the work done against the buoyancy force associated with bubble entrainment. Lamarre \& Melville (1991) found that up to half of the energy lost to the water 
column may be expended this way. However, they also found that the proportion of maximum volume entrained versus total energy lost due to breaking scales with the characteristic variables of the breaking wave. This implies that we can absorb these effects into the constant of proportionality, $\Xi$, relating circulation and wave energy dissipation in (3.18), just as they are included in the dissipation data of figure 1 . Also, breaking waves will generate both currents and waves (Bühler 2007); however, these waves were measured in the case of a plunging breaking wave by Rapp \& Melville (1990, §3.5) and found to contain less than $1 \%$ of the energy of the main packet. Furthermore, the contributions of irrotational waves to the contour integral of the instantaneous forcing function in (2.6) is zero. We note that although these effects were not explicitly taken into account in the scaling argument of Drazen et al. (2008), these phenomena are inherent in the laboratory studies used to corroborate the scaling model, shown in figure 1 (see also Romero et al. 2012).

Measurements of dissipation due to turbulence associated with breaking waves in the field are now available (see, for instance, Romero et al. 2012; Sutherland et al. 2012), so that using the results in this paper one could potentially estimate the circulation imparted to the underlying surface currents. Also of interest for application in the field is the description of the vertical vorticity at the surface, which can now be measured in the ocean (Veron, Melville \& Lenain 2009; Sutherland et al. 2012), and has been hypothesized to seed the so-called CL II instabilities leading to Langmuir circulations (Csanady 1994, SMM2007), an important feature of upper ocean dynamics. We note that in the absence of outside forcing, the circulation predicted by (3.1) is invariant along the core. This implies that surface signatures of vorticity can be proxies for the vorticity and mixing at depth, a result which could be used to corroborate the estimate of generated circulation based on the energy dissipated by breaking waves.

\section{Acknowledgements}

This research was supported by grants from the Office of Naval Research (Code 322) and the National Science Foundation (Physical Oceanography) to W.K.M. We thank the anonymous referees for comments that have lead to significant improvements to the paper; D. Drazen for providing readily accessible archived data from Drazen \& Melville (2009); B. Reineman for his assistance in generating some of the figures, and L. Romero for providing the template for figure 1 .

\section{REFERENCES}

Andrews, D. G. \& MCInTyRe, M. E. 1978 An exact theory of nonlinear waves on a Lagrangian mean flow. J. Fluid Mech. 89, 609-646.

BANNER, M. L. \& PEIRSON, W. L. 2007 Wave breaking onset and strength for two-dimensional deep-water wave groups. J. Fluid Mech. 585, 93-115.

Banner, M. L. \& Peregrine, D. H. 1993 Wave breaking in deep water. Annu. Rev. Fluid Mech. 25, 373-397.

Batchelor, G. K. 1967 An Introduction to Fluid Mechanics. Cambridge University Press.

BÜHLER, O. 2007 Impulsive fluid forcing and water strider locomotion. J. Fluid Mech. 573, 211-236.

Craik, A. D. D. \& Leibovich, S. 1976 A rational model for Langmuir circulations. J. Fluid Mech. 73, 401-426.

CSANAdy, G. T. 1994 Vortex pair model of Langmuir circulation. J. Mar. Res. 52, 559-581.

DhANAK, M. R. \& De BERnARdinis, B. 1981 The evolution of an elliptic vortex ring. J. Fluid Mech. 109, 189-216. 
Drazen, D. A., Melville, W. K. \& Lenain, L. 2008 Inertial scaling of dissipation in unsteady breaking waves. J. Fluid Mech. 611, 307-332.

Drazen, D. A. \& Melville, W. K. 2009 Turbulence and mixing in unsteady breaking surface waves. J. Fluid Mech. 628, 85-119.

DUNCAN, J . H. 1981 An experimental investigation of breaking waves produced by a towed hydrofoil. Proc. R. Soc. Lond. Ser. A 377, 331-348.

Duncan, J. H. 2001 Spilling breakers. Annu. Rev. Fluid Mech. 33, 519-547.

HASSELMANN, K. 1974 On the spectral dissipation of ocean waves due to white capping. Boundary-Layer Met. 6, 107-127.

Helmholtz, H. 1858 Uber Integrale der hydrodynamischen Gleichungen welche den Wirbelbewegungen entsprechen. J. Reine Angew. Math. 55, 25-55.

Hornung, H. G., Willert, C. \& Turner, S. 1995 The flow field of a hydraulic jump. J. Fluid Mech. 287, 299-316.

Kundu, P. K., Cohen, I. M. \& Dowling, D. R. 2012 Fluid Mechanics, 5th edn. Academic.

Lamarre, E. \& Melville, W. K. 1991 Air entrainment and dissipation in breaking waves. Nature 351, 469-472.

LAMB, H. 1932 Hydrodynamics. Cambridge University Press.

Leibovich, S. 1983 The form and dynamics of Langmuir circulations. Annu. Rev. Fluid Mech. 15, 391-427.

Lighthill, J. 1978 Waves in Fluids. Cambridge University Press.

Linden, P. F. \& TURner, J. S. 2001 The formation of optimal vortex rings, and the efficiency of propulsion devices. J. Fluid Mech. 427, 61-72.

Longuet-Higgins, M. S \& TuRner, J. S. 1974 An entraining plume model of a spilling breaker. J. Fluid Mech. 63, 1-20.

Melville, W. K. 1994 Energy dissipation by breaking waves. J. Phys. Oceanogr. 24, 2041-2049.

Melville, W. K. 1996 The role of wave breaking in air-sea interaction. Annu. Rev. Fluid Mech. 28, 279-321.

Melville, W. K. \& Rapp, R. J. 1985 Momentum flux in breaking waves. Nature 317, 514-516.

Melville, W. K., Veron, F. \& White, C. J. 2002 The velocity field under breaking waves: coherent structures and turbulence. J. Fluid Mech. 454, 203-233.

Peregrine, D. H. 1998 Surf zone currents. Theor. Comput. Fluid Dyn. 10, 295-310.

PEREGRINE, D. H. 1999 Large-scale vorticity generation by breakers in shallow and deep water. Eur. J. Mech. (B/Fluids) 18, 403-408.

Phillips, O. M. 1977 The Dynamics of the Upper Ocean, 2nd edn. Cambridge University Press.

PHILliPS, O. M. 1985 Spectral and statistical properties of the equilibrium range in wind-generated gravity waves. J. Fluid Mech. 156, 505-531.

Pope, S. B. 2000 Turbulent Flows. Cambridge University Press.

RAPP, R. J. 1986 Laboratory measurements of deep water breaking waves. PhD thesis, Ocean Engineering, Massachusetts Institute of Technology.

RApp, R. J. \& Melville, W. K. 1990 Laboratory measurements of deep-water breaking waves. Phil. Trans. R. Soc. Lond. A 331, 735-800.

Restrepo, J. M., Ramirez, J. M., MCWilliams, J. C. \& Banner, M. 2011 Multiscale momentum flux and diffusion due to whitecapping in wave-current interactions. J. Phys. Oceanogr. 41, 837-856.

Romero, L., Melville, W. K. \& Kleiss, J. 2012 Spectral energy dissipation due to surface-wave breaking. J. Phys. Oceanogr. 42, 1421-1444.

Saffman, P. G. 1992 Vortex Dynamics. Cambridge University Press.

Shariff, K. \& LeOnARd, A. 1992 Vortex rings. Annu. Rev. Fluid Mech. 24, 235-279.

Sullivan, P. P., McWilliams, J. C. \& Melville, W. K. 2004 The oceanic boundary layer driven by wave breaking with stochastic variability. Part 1. Direct numerical simulations. J. Fluid Mech. 507, 143-174.

Sullivan, P. P., McWilliams, J. C. \& Melville, W. K. 2007 Surface gravity wave effects in the oceanic boundary layer: large-eddy simulation with vortex force and stochastic breakers. J. Fluid Mech. 593, 405-452. 
Sutherland, P., Melville, W. K., Lenain, L. \& Statom, N. 2012 Measurements of near-surface wave coherent turbulence in the presence of breaking waves. The Oceanogr. Soc., the Am. Soc. of Lim. and Oceanogr. and the Am. Geophys. Union 2012 Ocean Sci. Meet., Abstract \# 10699.

TAIT, P. G. 1867 Translation of (Helmholtz 1858): On the integrals of the hydrodynamical equations, which express vortex-motion. Phil. Mag. 33, 485-512.

TAYLOR, G. I. 1953 Formation of a vortex ring by giving an impulse to a circular disk and then dissolving it away. J. Appl. Phys. 24, 104.

Thorpe, S. A. 2005 The Turbulent Ocean. Cambridge University Press.

Tian, Z. G., Perlin, M. \& ChOI, W. 2010 Energy dissipation in two-dimensional unsteady plunging breakers and an eddy viscosity model. J. Fluid Mech. 655, 217-257.

Veron, F., Melville, W. K. \& Lenain, L. 2009 Measurements of ocean surface turbulence and wave-turbulence interactions. J. Phys. Oceanogr. 39, 2310-2323.

White, C. J. 1996 A laboratory study of breaking waves using digital particle image velocimetry. MS thesis, University of California, San Diego. 